\title{
Development and application of an off-site maintenance system in the petrochemical industry
}

(C) The Author(s) 2017. Published by Higher Education Press. This is an open access article under the CC BY license (http:// creativecommons.org/licenses/by/4.0)

\begin{abstract}
On-site maintenance (ONSM) is critical to ensuring the safety of equipment in the petrochemical industry. However, many accidents occur during ONSM processes because of unstable equipment, complicated work environment, and human error. To reduce congestion and exposure to hazards during on-site operations and thereby reduce the probability of accidents, off-site maintenance (OFSM) is proposed based on Energy Release Theory (ERT). The information requirements for OFSM are analyzed. A support system for OFSM, which makes use of visualization, information management, and localization capabilities, is developed. A case study utilizing OFSM and its support system for an oil-loading facility of a petrochemical company is conducted. The application results indicated that implementation of OFSM system can significantly reduce the operation risks and can improve the operation efficiency.
\end{abstract}

Keywords on-site maintenance, petrochemical industry, safety, off-site maintenance system

\section{Introduction}

Safety is one of the most significant challenges in the petrochemical industry, and it is critical to the success of a petrochemical operation. However, in the said industry, the frequency of accidents has significantly increased in the last decades (Fabiano and Curro, 2012). The petrochemical

Received April 26, 2017; accepted May 22, 2017

Ruisheng YONG

Guangxi Petrochemical of China Petroleum, Qinzhou 535000, China

Yanbing YE, Hanbin LUO (ه), Lieyun DING

School of Civil Engineering and Mechanics, Huazhong University of Science and Technology, Wuhan 430074, China

E-mail: luohbcem@hust.edu.cn industry has been regarded as one of the major high-risk industries worldwide because of the high-risk characteristics of petrochemical installations (Nivolianitou et al., 2006), which include the following: (1) the operation involves complex processes with processed flammable and explosive substances; (2) losses would be unimaginable once there is a major unpredictable failure of an establishment (Guo et al., 2009); (3) the consequences can affect not only many people inside and outside these establishments, but also the surrounding environment (Papazoglou et al., 1999); (4) inherent congestion in process installations, whereby process equipment is often situated in close proximity to one another, increases the probability of catastrophic accidents and amplifies their potential consequences (Baesi et al., 2013).

To ensure that petrochemical installations are in good operational condition, a large part of the workforce and workers have to perform equipment maintenance work, some of which are the most hazardous activities (Waeyenbergh and Pintelon, 2002). Petrochemical equipment maintenance is more dangerous compared to maintenance in other industries. A significant proportion of serious accidents occurring in the petrochemical industry are related to maintenance. Accidents are more prone to occur during maintenance processes than in normal operating circumstances. According to Chinese statistics, of all major accidents occurring in the Sinopec Group, accidents due to equipment maintenance processes accounted for $42.6 \%$ (Liu, 2003). In the last decade, nearly $70 \%$ of the accidents in PetroChina Southwest Company occurred during their equipment maintenance processes (Yuan and Guo, 2011). This status is in good agreement with a survey on the reported incidents in the Greek Petrochemical Industry, where the maintenance phase represents the second highest percentage $(15 \%)$ of the total number of incidents (Konstandinidou et al., 2006). Consequently, safety has become one of the major issues in petrochemical equipment maintenance management. 
1.1 Maintenance management in the petrochemical industry

Maintenance can be broadly classified into two types: corrective maintenance $(\mathrm{CM})$ and preventive maintenance (PM) (Li et al., 2006). Some researches have focused on selecting the optimal maintenance policies or actions as a routine to be undertaken for improving components at the lowest expected cost (Murthy and Asgharizadeh, 1999; Sherwin, 2000; Adolfo and Heguedas, 2002). While all these models focus on maintenance cost (as an objective or a constraint), several researches suggested that other factors should be taken into account when selecting a maintenance policy. For example, Waeyenbergh and Pintelon (2009) emphasized that implementation of a customized maintenance concept should offer possibilities to focus on other aspects, e.g., improved workplace safety. Martorell et al. (2005) applied an approach integrating reliability, availability, maintainability, and safety (RAMS) to decision-making of a multi-objective maintenance optimization. Li (2013) developed an equipment maintenance and safety integrity management system to determine the risk state of an equipment timely and accurately and to optimize maintenance schedules.

However, despite the fact that some current maintenance strategies have considered safety, they just focus on controlling failure and deterioration through timely and corrective equipment maintenance to ensure safety of the work environment. First, whatever maintenance strategies (i.e., CM or PM) are carried out, maintenance work (repairing the defect or replacing with new equipment) is inevitable. Second, equipment failure and maintenance corrective actions cannot be avoided completely while preventive maintenance strategies are applied because of the stochastic nature of equipment failure (Wang et al., 2007). Moreover, "breakdown maintenance" (which accounts for almost 50\%) and conventional TBM (which accounts for almost 35\%) are still dominant in Chinese petrochemical enterprises (Cong, 2013). Unfortunately, current maintenance management is paying little attention to the safety of these maintenance activities (repair and replacement of equipment). In fact, these maintenance processes are always the major cause of various accidents as mentioned above.

\subsection{Safety challenges of ONSM in petrochemical industry}

Maintenance work that is commonly performed directly at the production site is regarded as on-site maintenance (ONSM) in this study. A typical ONSM process (Fig. 1) consists of the main team members (in boxes) and the types of activities and processes that might be conducted (arrows linking these boxes). To improve the safety performance during ONSM processes, several solutions have been proposed, which include the following:

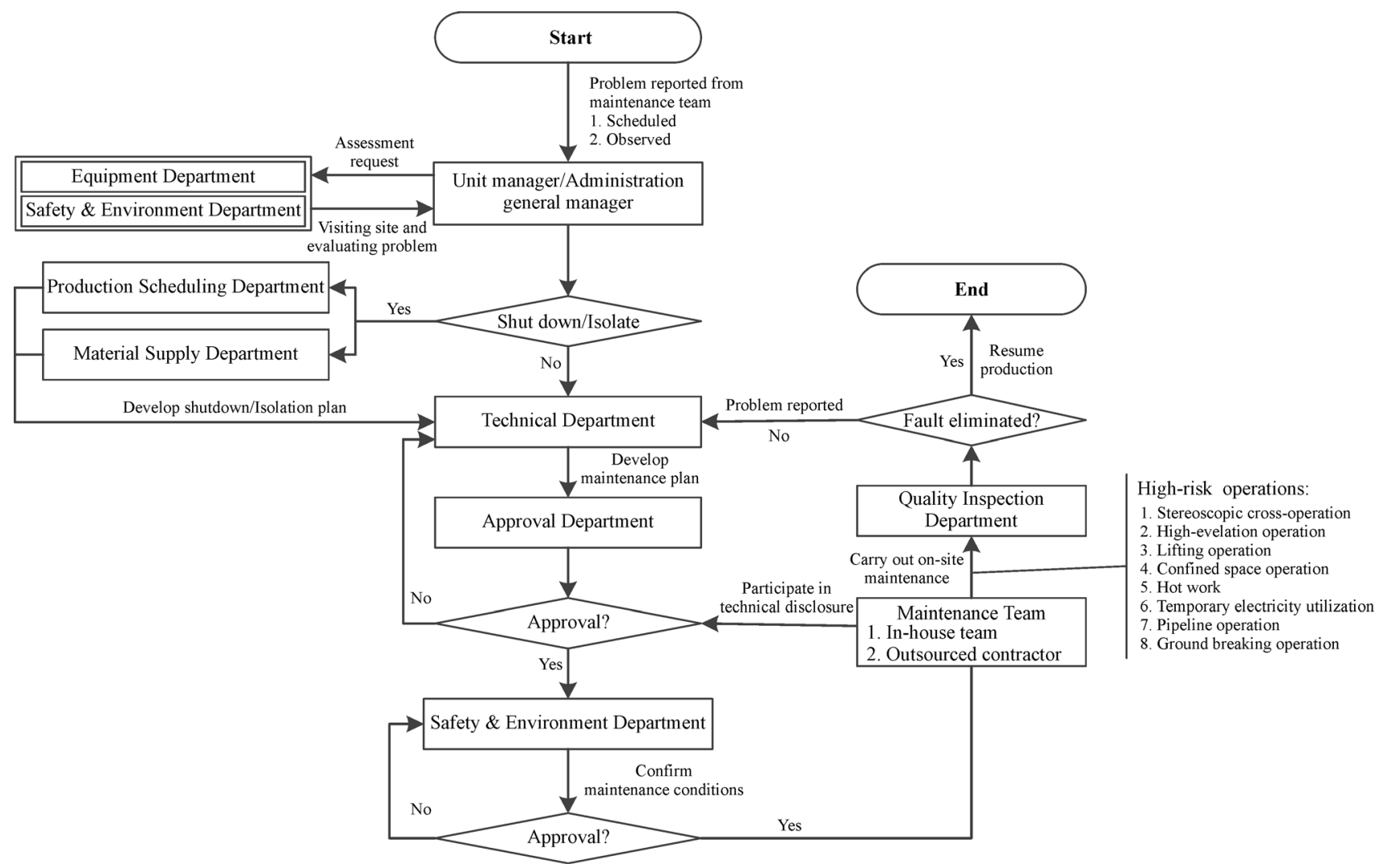

Fig. 1 A typical ONSM process 
(1) legislations concerning safety and health; these have been instituted in many countries and regions, e.g., a total of approximately 300 chemical safety control regulations and more than 600 national safety standards have been promulgated in China during the past decade (Xue and Zeng, 2010);

(2) techniques such as hazard and operability assessment, fault tree analysis, and layer of protection analysis; these have been developed and are widely applied to identify and control potential hazards by materials and conditions (Knegtering and Pasman, 2009);

(3) organizational management approaches, safety plans, and communication procedures; these have been proposed to increase protection consciousness and reduce conflicts and accidents (Ben-Daya et al., 2009).

These types of approaches have been applied for many years, and several hazardous, out-of-control conditions are identified and prevented as a result. However, there are still numerous accidents during ONSM processes today (Knegtering and Pasman, 2009; Zhao et al., 2013).

Accidents generally result from an unsatisfactory interaction of human factors and growing complexity of equipment and environment (Attwood et al., 2006). During ONSM processes, safety challenges are briefly identified as follows:

(1) Unstable equipment. A wide range of complex equipment and facilities with their flammable, explosive, and toxic process media are usually handled at elevated temperatures and/or pressures, leading to inherent danger of petrochemical equipment (Zhang and Ye, 2010). Accidents are prone to occur because of the inherently vulnerable characteristics of petrochemical equipment, in addition to the implementation of high-risk operation (e.g., hot work) during ONSM processes.

(2) Complicated work environment. On-site work environment consists of several uncertainties that include 1) flammable and explosive media in air or around maintenance areas increasing the risk of fire and explosion; 2) narrow operation areas make stereoscopic crossoperation, high-elevation operation, and lifting operation prone to mechanical injuries, falling accidents, lifting injuries, etc.; and 3) sometimes, bad weather conditions also bring danger to maintenance works (Zhang, 2012).

(3) Human error. The performance of maintenance tasks is usually done with high intensity and is carried out on a tight schedule. Personnel engaged in maintenance works are not always familiar with the equipment, safety procedures, and on-site environment. These circumstances may cause unstable psychological (e.g., stress, fear, and scatterbrain) and physiological fatigue to maintenance workers, resulting in a lack of safety competency and increased likelihood of operational mistakes (Ishekwene, 2012).

The causes that may have contributed to industrial accidents have been presented in several studies and reports, e.g., the accident at Esso Australia's gas plant at
Longford, Victoria in September 1998 (Skogdalen and Vinnem, 2012), an analysis of UK petroleum refinery incidents (Luan, 2011), and a survey on accidents in the downstream oil industry from the early 1930s to 2010 (Fabiano and Curro, 2012). However, different causes can be identified by different accident investigators even from the same evidence. Moreover, only the immediate causes are often found (Wang, 2003) as it is difficult to determine the underlying causes because of the interactive effects of unstable equipment, complicated work environment, and human error during ONSM processes. Accidents may be more likely to occur when a maintenance worker works on site for a long time. Therefore, we should look for ways of avoiding hazards fundamentally. As a result, petrochemical equipment maintenance processes require a paradigm shift in the way they are operated, instead of the existing conventional ONSM practices. There is a need to develop strategies for eliminating the dangerous effects of complicated work environment and unstable equipment, and for the optimization of human performance and minimization of human errors.

1.3 The theory of accidental release of energy and implication

Developing from the physical nature of accidents, the theory of accidental release of energy expounds the process of the accidents: The unsafe behavior of operators and the insecure state of equipment caused by management errors as well as their interaction, give rise to abnormal or undesirable energy release of dangerous substances, which will make an effect on human bodies and facilities, causing casualties and property damage. Accidents can be prevented by reducing energy and strengthening shielding. If energy which is indispensable in people's life and the production process is out of control, energy release or escape will occur against the will of human beings, which may terminate the ongoing activities and bring about accidents, resulting in accident casualty or property loss.

According to the energy release theory, under the influence of the above-mentioned risk factors, unstable equipment, complex environmental conditions and maintenance personnel's errors will lead to risk events. For example, with multi-factors jointly reacting, unstable equipment may release energy. At the same time, maintenance staff exposed to this energy will be injured when the energy value exceeds what the maintenance staffcan bear. Similarly, other facilities in the same place likely to release energy are also exposed to this energy, and when the energy value exceeds what they can bear, a risk loss will arise.

In this study, an off-site maintenance (OFSM) model and its support system are developed to improve the safety performance during petrochemical equipment maintenance processes. Some other applications of off-site operations along with their benefits are mentioned, and the OFSM 
process model is described in Section 2. The information requirements for OFSM are identified, and the conceptual framework of applying this system is developed and these are presented in Section 3. The overall architecture and functional modules of the proposed system are discussed in Section 4. The benefits of implementing the OFSM system are demonstrated through a practical maintenance operation of an oil-loading facility, which is discussed in Section 5. Finally, the findings of this study are summarized in Section 6.

\section{Off-site maintenance}

Many researchers and practitioners have reported the benefits of off-site operations. Moghadam et al. (2012) stated that a factory-based modular construction process can increase the speed of construction and improve quality and safety. Ikuma et al. (2011) suggested that off-site operations have the potential advantages in safety over onsite construction, because they can be performed under a controllable environment and standardized processes. In an off-site construction of an extraordinary building, Maas and van Eekelen (2004) found that the weather has little direct influence on the construction (usually, quality is influenced by weather conditions). After conducting a detailed investigation on five building projects using a precast construction method, Jaillon and Poon (2009) declared that off-site prefabrication contributed to a cleaner and safer work environment on site, and also a reduction of construction time at a later stage of the project as less finishing works were required to be completed on site. Gibb and Isack (2003) pointed out that through the minimization of on-site operations and congestion, improved health and safety along with higher and more predictable quality are identified. Rodriguez et al. (2009) considered that while conducting intensive operation with the possibility of exposure to hazards for a prolonged time, health and safety would be improved by removing the operators from the immediate work area. The advantages of OFSM over ONSM are summarized in Table 1.

The above-mentioned applications on construction industry demonstrated the potential of off-site operations to improve the safety performance. Petrochemical equipment maintenance requires a transformation of operation mode from on-site operation to off-site operation to reduce accidents fundamentally. Therefore, the development and application of OFSM in the petrochemical industry are proposed. To achieve this objective, the implementation process of OFSM is developed first, as shown in Fig. 2.

The objective of this proposed OFSM model is to reduce the safety risk during petrochemical equipment maintenance processes, improving the safety performance and protecting the staff, public, and environment. OFSM carries out real-time maintenance away from the production area based on an observed incipient failure signal or a planned schedule. Once the need for maintenance is identified, the fault is evaluated to make a decision on whether to shut down the production facility or isolate the faulty equipment. Then, the faulty equipment is dismantled and delivered to the maintenance workshop for off-site repairs. The workshop is built based on the distribution of production facilities and the division of maintenance business, and is located outside the production area. The repairs are undertaken in the workshop. After the detection test and ensuring that faults have been eliminated, the equipment is reinstalled to resume production.

The core of OFSM is to perform off-site prefabrication operation as much as possible, which means that the operation of piping, welding, and installation shall be carried out in a safe and controllable maintenance workshop and finally, integral reinstallation shall be implemented on site. By shifting more and more conventional on-site operations undertaken in a ground maintenance workshop, OFSM reduces the congestion, exposure to hazards, and high-risk operations, e.g., stereoscopic cross-operation, high-elevation operation, confined space operation, and hot work (Fig. 2 ). The workshop provides all types of maintenance tools, which are kept in order based on functional classification. The workers can easily find the tools they want. The repairs can be undertaken by complying with a preplanned work schedule. The improvement of the work environment and the lesser finishing operations required to be completed on site also decrease the work intensity and pressure. In addition, it is easier to supervise and control the safe maintenance operation in a workshop. All of these reduce the propensity for human mistakes and further improve the work efficiency and safety. Consequently, under a controllable work environment along with (1) the minimization of

Table 1 Comparative analysis between ONSM and OFSM (Moghadam et al., 2012; Ikuma et al., 2011; Maas and van Eekelen (2004); Jaillon and Poon, 2009; Gibb and Isack, 2003; Rodriguez et al., 2009; Wong et al., 2010)

\begin{tabular}{lccc}
\hline Items & On-site operation & Off-site operation & Benefits description \\
\hline Work environment & On-site, field operation & Off-site, workshop operation & Controllable work environment \\
Influence of external factors & Affected significantly by external factors & Affected slightly by external factors & Improvement of maintenance quality \\
High-risk operation & More operation types and workload & Less operation types and workload & Reduction of risk \\
On-site duration & Long & Short & Reduction of exposure to hazards \\
Implementation & Multi-point operation, multi-task interface & Standardized operations & Better safety management \\
\hline
\end{tabular}




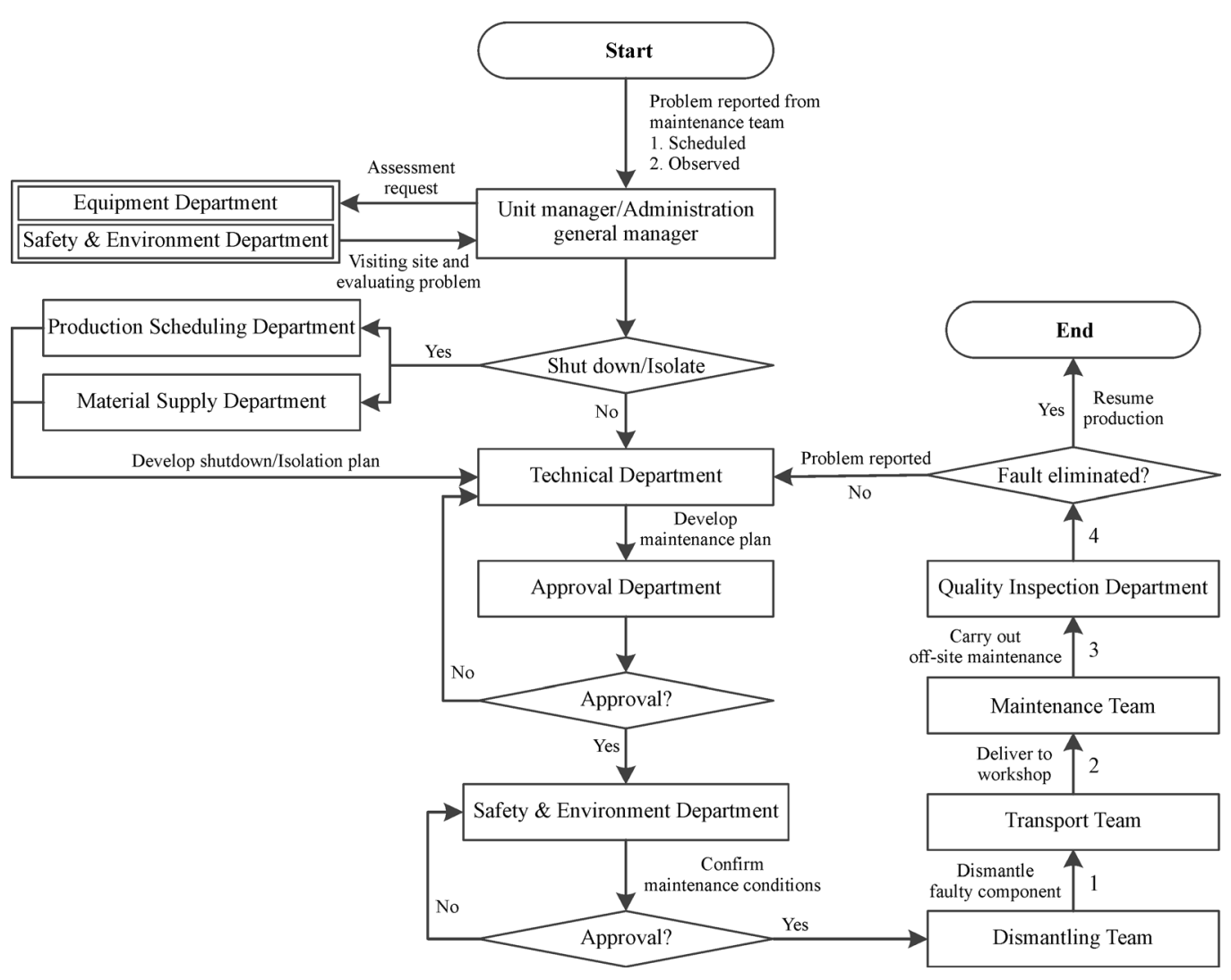

Fig. 2 Flow chart of OFSM model

on-site operations, (2) less congestion, and (3) reduction of exposure to hazards, we believe that OFSM will be safer than the conventional ONSM.

\section{OFSM-based support system}

\subsection{Information requirements analysis}

To successfully utilize this maintenance strategy, the requirements for its implementation should be clearly identified. A successful application of such OFSM practice could be jeopardized owing to a lack of sufficient information on the equipment available for any maintenance operation (Motawa and Almarshad, 2013). This is because maintenance is composed of a set of activities where it is very difficult to find procedures and information support systems in one place to facilitate the improvement process (Vagliasindi, 2003). Lack of equipment, process knowledge, and historical data to complete the required analysis in time are recognized as the main constraints in implementing a maintenance operation (Hipkin and De, 2000). In addition, with information scattered around in many different stakeholders (e.g., design team, construction team, and maintenance management team), poor information communication has often made the maintenance staff frustrated during operation. In practical terms,
OFSM processes involve the requirements for information capture, reuse, and sharing all throughout the entire maintenance process.

As information requirements and expectations are important for OFSM (Fig. 2), the opportunities and mechanisms for information capture, reuse, and sharing were identified. The first opportunity exists in "Process 1 ," where the dismantling team dismantles a large plant into equipment components (e.g., a large number of small items such as valves and small pumps) under the guidance of the original design and installation files, information of upstream control device, and other relevant operating procedures. The second opportunity is found in "Process 3 ," when the maintenance team conducts the operation within the OFSM workshop. In this process, the workers need a full understanding of the maintenance objective to carry out an effective operation with the information support, e.g., equipment ledger information, routine maintenance records, knowledge of failures and accidents, and maintenance program. The third opportunity exists in "Process 4," when the quality inspection department carries out the detection test under the guidance of acceptance standards and requirements. The fourth opportunity takes place in "Process 5," when the installation team performs the reinstallation operation. This process requires information for locating the various components to ensure that reinstallation is in accordance with the 
original plant structure. The information requirements for OFSM are illustrated in Fig. 3.

To state in detail, the information required and their specific applications to support a smooth and effective implementation of OFSM are as follows:

(1) Requirement 1 (R1): Quick access to information to identify the need for maintenance and to deal with equipment faults during an emergency; fast retrieval of applicable petrochemical plant design and installation files to guide the dismantling team.

(2) Requirement 2 (R2): Comprehensive information for the maintenance workers to develop an overall understanding of the equipment components owing to a lack of scene perception in the OFSM workshop; rapid retrieval and capture of attributes and maintenance information on each scattered equipment component.

(3) Requirement 3 (R3): Quick access to information to carry out the detection test.

(4) Requirement 4 (R4): Information to locate various scattered equipment components, and to facilitate an efficient decision-making in conducting the reinstallation operation.

\subsection{Off-site maintenance system}

A successful implementation of maintenance requires a fast acquisition of high-quality information on the faulty equipment. However, the information is normally spread across multiple disciplines and restricted groups within organizations (e.g., design team, construction team, and maintenance management team). Data are continuously transferred between systems in organizations, which reduces their clarity and accuracy owing to the lowest common denominator for sharing information across the asset management systems. These isolated structures contain disconnected data formats that simply cannot communicate with each other and cannot be shared to enable a uniform and consistent access and updates. The ability to bring together all these information from an individually connected set of applications is seldom presented in most operation and maintenance strategies today. Particularly for OFSM, to meet the requirements (i.e., R1-R4) and allow the operator to perform the maintenance effectively, what is needed is not only a full representation of the digital asset that contains the complete engineering and operational information, but also a visualized presentation of information that is associated with spatial equipment entity.

AVEVA's information management and engineering design solutions have been used to create a powerful and digital as-built plant in the petrochemical industry, delivering accurate, complete, and accessible information throughout the asset life cycle. Using AVEVA PDMS (plant design management system), the logical model of a plant can be quickly and efficiently modeled into a full 3D design layout.

The created virtual 3D model with properties accurately reflects the as-built facility and also forms the basis of a common digital infrastructure for all as-built information and deliverables. All the associated plant information, such as drawings, P\&IDs (piping and instrumentation diagrams), maintenance reports, procedures, photographic data, documents, and any other datasets, are connected to the corresponding 3D design model. As a result, AVEVA provides a highly effective and intuitive $3 \mathrm{D}$ visualization tool for displaying and interacting with plant model data. Users can acquire a realistic and easily navigated view of the model and check equipment attributes, location, and

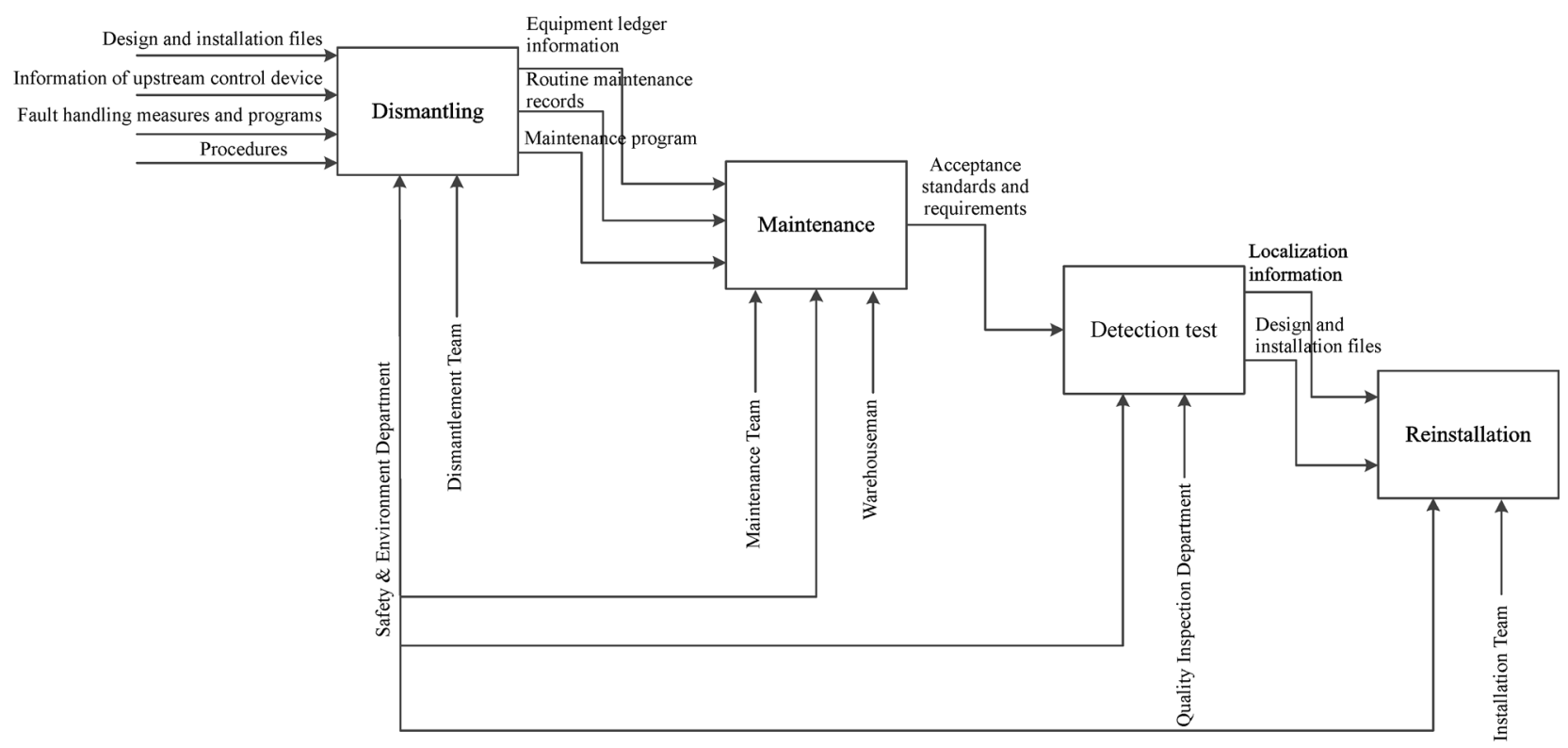

Fig. 3 Information requirements for OFSM 
status. In addition, AVEVA offers their users realistic and immersive 3D virtual worlds in which they can engage with other operators and with objects and entities that behave in a convincingly lifelike manner. The ability to interact with these virtual worlds in increasingly sophisticated ways has at last made it possible to create accurate simulations of real-world environment (AVEVA). Consequently, based on the AVEVA solutions, a 3D model visualization for maintenance can be developed to enhance the efficiency and safety of the petrochemical equipment OFSM. The application model of AVEVA PDMS integrated with information management solutions for OFSM is illustrated in Fig. 4.

Therefore, this research combined the AVEVA PDMS with information management solutions to develop an integrated OFSM system that enables the operators to perform OFSM more effectively and efficiently. Figure 5 illustrates the conceptual framework of applying this proposed system in OFSM. The system mainly supports OFSM practices with visualization, information management, and localization capabilities. First, as the 3D model manipulates the geometry, spatial relationships, geographic information, object attributes and specifications (Singh et al., 2011), the 3D model visualization provides users a better and more realistic understanding of the operational equipment and its associated information. Second, pieces of information from all sources, i.e., existing digital data and documents, paper documents, as-built surveys, and photographs are assembled and associated with corresponding 3D models. The system provides a database with complete and substantial information to reduce the operating risk owing to information fragmentation and loss. Third, the localization capability not only takes the user to the right maintenance object, but also makes all data and documents relating to it instantly accessible, through the associated engineering tag. The capabilities associated with the detailed description of the system are presented in Table 2 .

\section{System development}

\subsection{System architecture}

This section presents the establishment of a system for maintaining a visualized information to assist the OFSM in the petrochemical industry. The system application relies on a seamless interconnection between the 3D model base and information base through the AVEVA PDMS integrated with information management solutions, as shown in Fig. 6. The 3D model base provides visual models of the petrochemical plant, equipment components, site facilities, etc. The information base stores the entire life cycle data of these plants and equipment. Both of these

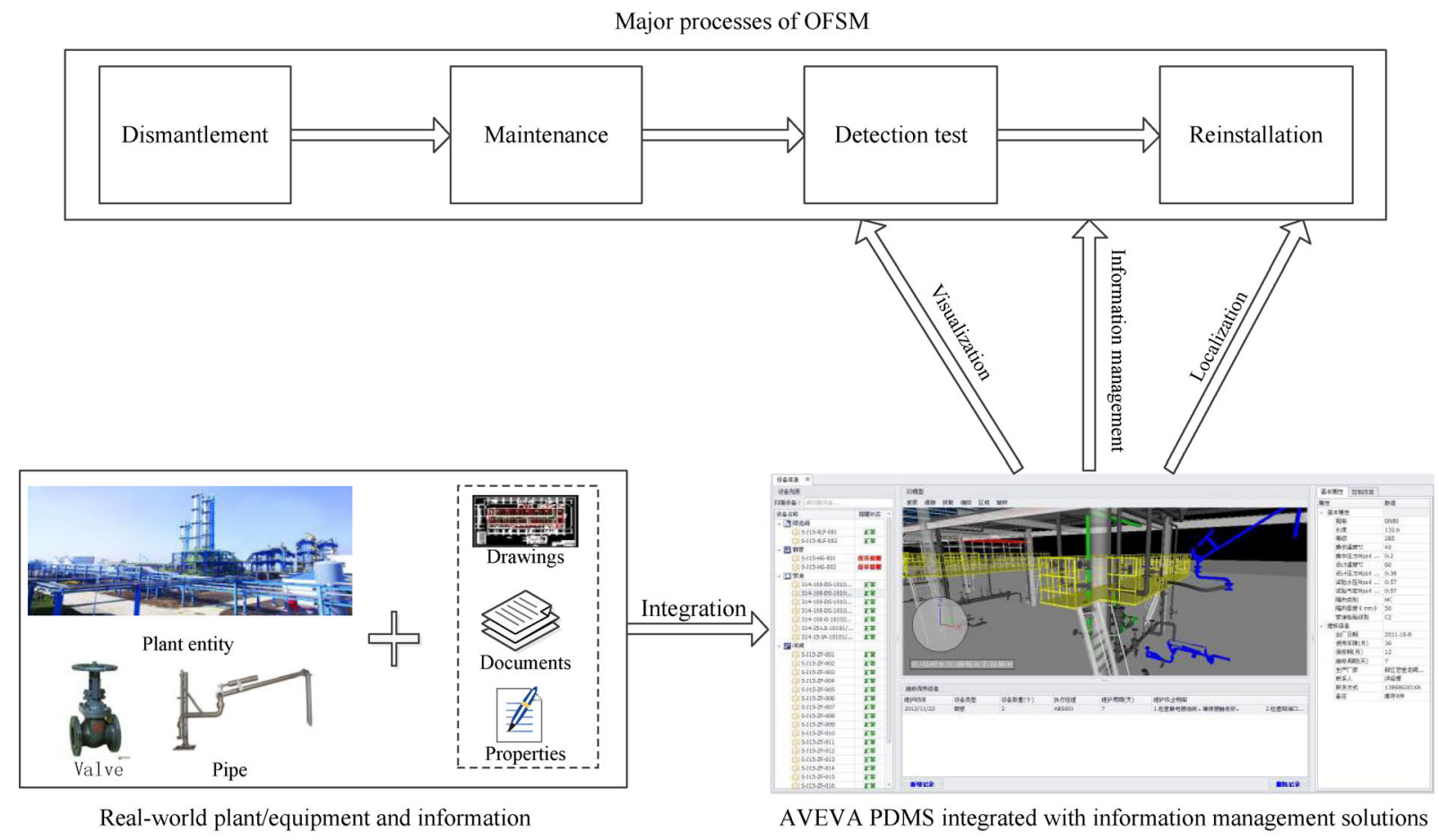

Fig. 4 The application model of AVEVA solutions for OFSM 


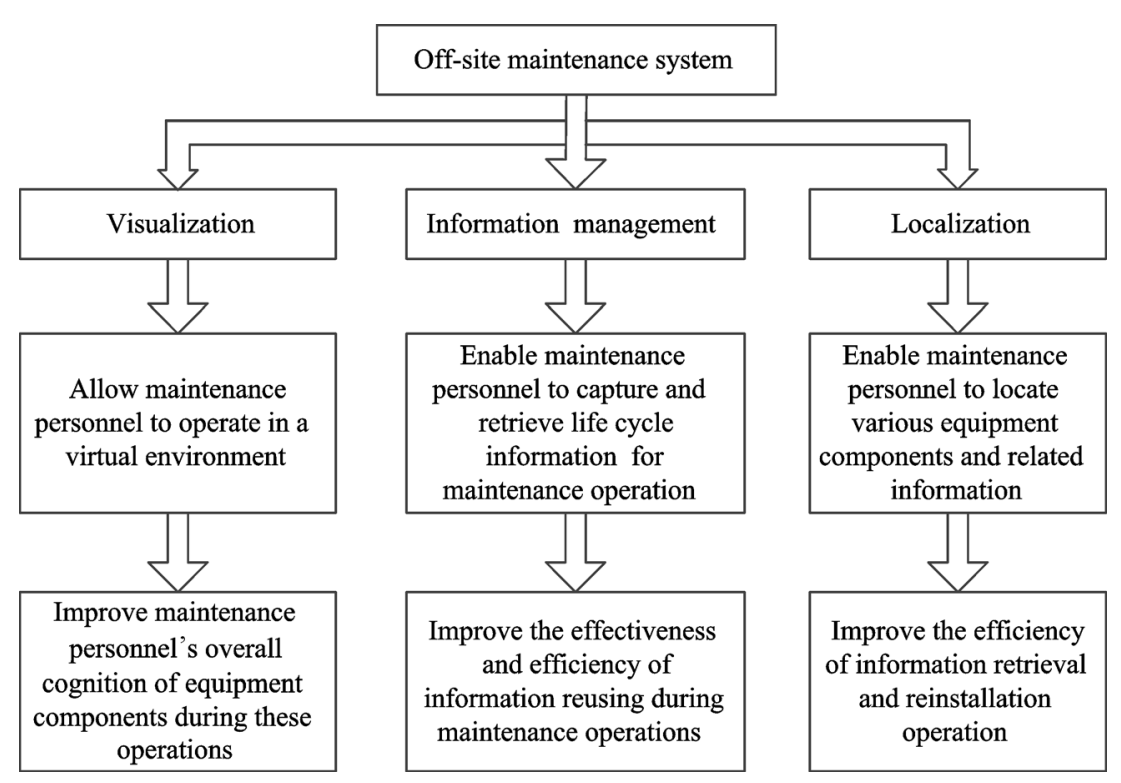

Fig. 5 The conceptual framework of applying off-site maintenance system

Table 2 Capabilities of off-site maintenance system

\begin{tabular}{ll}
\hline Capabilities & \multicolumn{1}{c}{ Description } \\
\hline Visualization & - Provide a virtual environment of plant/equipment similar to the real world \\
& - Facilitate the understanding of information that are attached to the corresponding equipment components \\
Information management & - Facilitate identification of dismantling and reinstallation sequences \\
& - Provide complete, accurate, and accessible information for maintenance operators \\
Localization & - Enable accurate identification of maintenance object and fast capture and retrieval of related information \\
& - Facilitate reinstallation operation with spatial coordinates localization \\
\hline
\end{tabular}

bases are automatically associated and provide the data resource for OFSM. Furthermore, the internet/intranet interaction platform provides convenience for collaboration among all relevant disciplines, contributing to a better information communication and sharing. At the domain layer, all types of capability applications (i.e., visualization, information management, and localization) are realized.

\subsection{Visualization module}

The visualization module is designed to provide a virtual environment by generating parametric 3D digital objects that represent real-world (physical) plant/equipment. Visualized through the intuitive graphical interface, the user can quickly identify the equipment that should be maintained through a "maintenance warning" function. By clicking on the equipment, the user can instantly acquire a list of other documents and data in which the object is referenced. For instance, the "maintenance procedures" can help the user to deal with an equipment fault in an emergency. Through the "video instructions," the user can become familiar with the on-site environment, identify safety risks, and understand the operating procedures prior to conducting dismantlement/reinstallation. When piles of scattered equipment components are delivered to the OFSM workshop, it is difficult for the workers to maintain a thorough understanding of the maintenance objects. The virtual "3D model review" allows the user to review the equipment in a panoramic $360^{\circ}$ view to obtain an overall understanding of its original appearance. With visualized 3D model and associated information (e.g., basic attributes, control information, and maintenance records), the workers can carry out the OFSM efficiently owing to the improved equipment familiarity.

\subsection{Information management module}

As mentioned above, while executing maintenance tasks, the workers always need a large amount of information, usually including various sources (e.g., digital data and paper document) that are formalized at different stages (e.g., engineering, construction, maintenance, and operation) across different disciplines. These isolated structures and disconnected data formats make it difficult to perform the maintenance operation efficiently. The OFSM system 


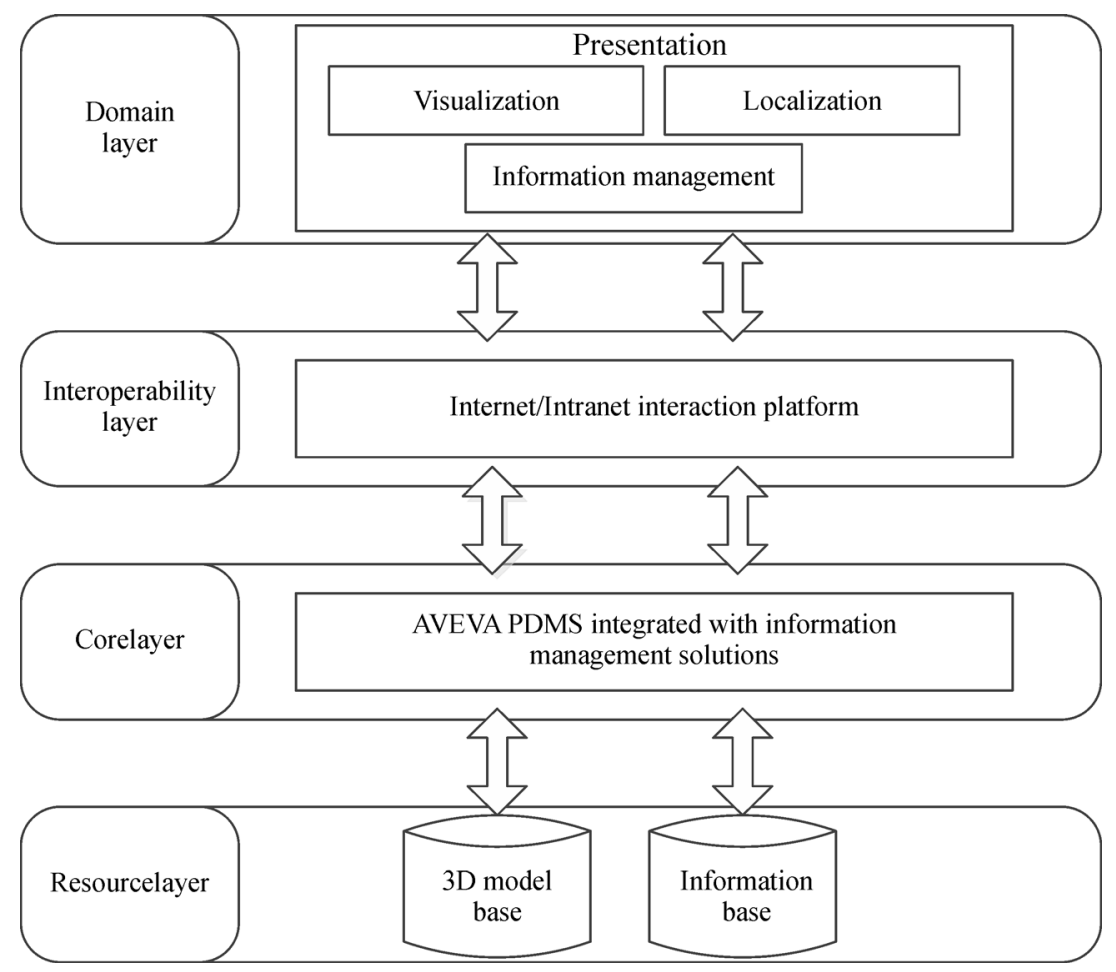

Fig. 6 The architecture of off-site maintenance system

developed in this study can provide a solution to this problem through an integrated information management module. The accurate information through the life cycle of the equipment is assembled into the module. The information management module is composed of equipment management, maintenance management, inventory management, risk management, and security/administration, as shown in Fig. 7.

By depicting non-geometric metadata and relationships among equipment components, a consistent object is described according to the model attached to it. A maintenance object contains the following information as listed in Table 3. The properties and characteristics of an object are represented by its attributes. The data (e.g., manufacturer/vendor information and location information) and documents (e.g., specifications, operation and maintenance manuals, manufacturing instructions, and test reports) are also integrated into the module. In addition to the information, professional maintenance knowledge gained during daily preventive maintenance operation, such as lessons learned from the investigation of failure causes, reasons for selecting a specific method of maintenance, selection of specialist contractors, and ripple effects on the other elements are also recorded in detail. All the information are transferable through the associated 3D models and remain dynamically updated in real time. Furthermore, the equipment is classified into groups according to category. Moreover, in accordance with the petrochemical industry standard, each piece of equipment is defined with a standard name and unique identification (ID) number. With the standardized integrity management of this information, the effectiveness and efficiency of maintenance can be improved.

To search for information on a specific maintenance object, the user can query by its category, name, ID, or identify it using a radio frequency identification (RFID) tag. Automatic identification of equipment and information can be achieved by applying RFID technology, which improves the equipment maintenance efficiency (ChienHo, 2009). Each maintenance object has a unique ID number that is written onto the RFID tag. An RFID reader is used to send a radio signal to the tag and read its response, and then the detailed data related to the object can be displayed in the computer system. For OFSM, the RFID tag is affixed to the corresponding equipment component. When a worker needs to query the information on a maintenance object, he/she can instantly acquire the visualized information in the OFSM system by using an RFID reader to read the tag.

\subsection{Localization module}

The main objectives of the localization module include the localization of upstream control equipment and the identification of maintenance object and its associated information. Once a faulty equipment has been identified, the workers need to know where the upstream control equipment is located. With the localization of upstream 


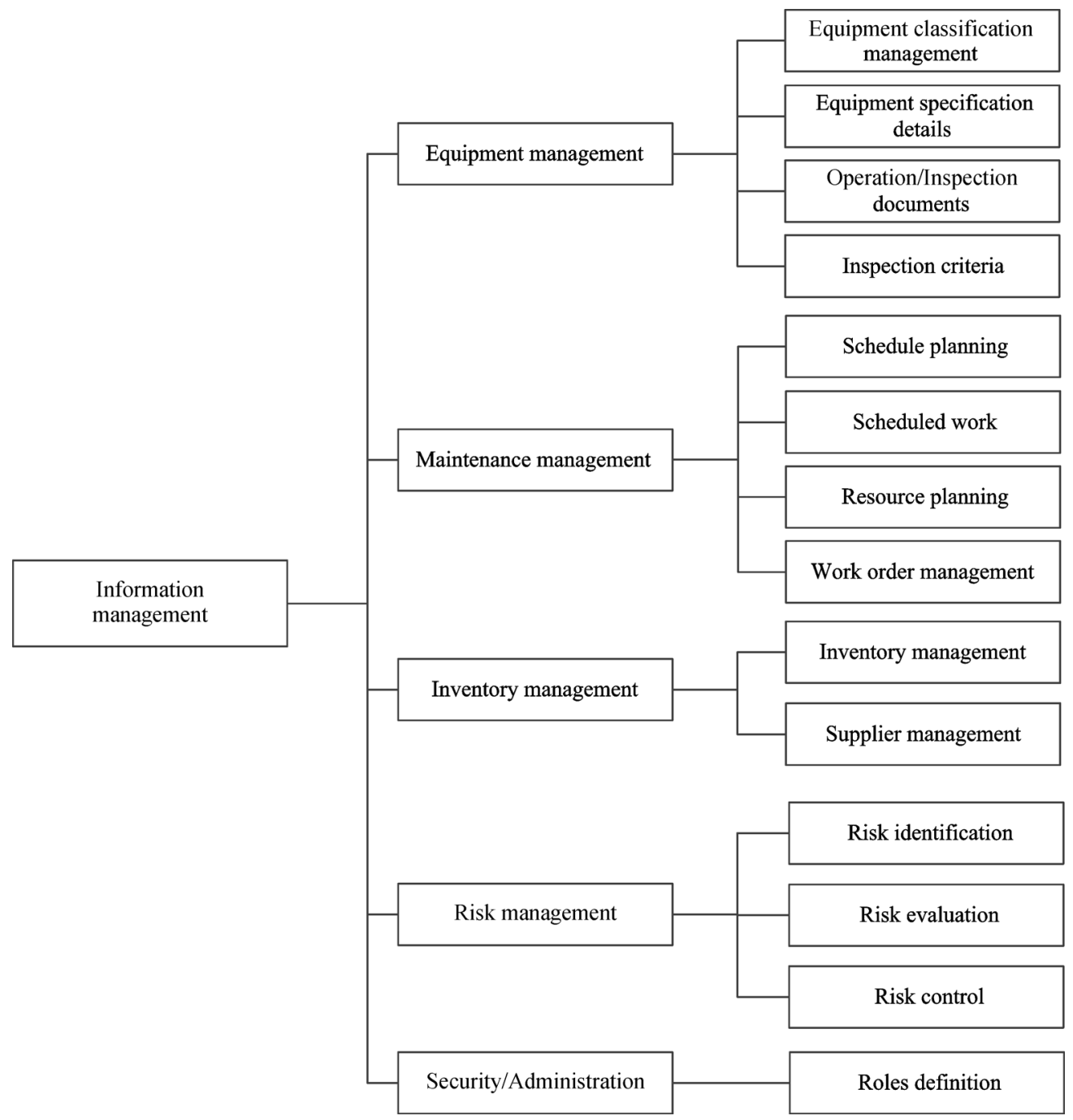

Fig. 7 Information management module configuration

Table 3 Classification of equipment information

\begin{tabular}{ll||ll}
\hline Object & Attribute & Object & Attribute \\
\hline Equipment & Name & Maintenance & Factory date \\
Records & Equipment category & Service life \\
& Equipment ID & Warranty period \\
& Size, length, depth & Maintenance date \\
& Material, medium & Duration \\
& Temperature, pressure & Maintenance cycle \\
& & Maintenance team, \\
Documents & Operating procedures & Maintenance works \\
& Inspection criteria & Risk factors \\
Laws and Regulations & Risk types \\
& Original as-built files & Failure causes \\
& Accident reports & Risk level \\
Lesson records & Risk control measures \\
& & Manufacturer/Vendor \\
\hline
\end{tabular}


control equipment, the workers can navigate into it and identify surrounding items in the 3D model interface. Equipment familiarity can be improved, and time can be saved when they take emergency control measures on site. While carrying out maintenance in the OFSM workshop, it is critical to quickly identify the scattered equipment components and gather the required information. Particularly, when the responsibility for the facility is taken over by newly assigned workers and/or an outsourced maintenance team, it is crucial to help them effectively obtain a comprehensive understanding of the equipment components. The localization module provides an efficient equipment localization and instant accessibility to relevant information through the associated RFID tag.

\section{Case study}

\subsection{Case background}

Guangxi Petrochemical Company, located in Qinzhou City, China, has 12 sets of oil refining production facilities. A large number of maintenance operations are carried out on site every year. However, accidents often occur during the ONSM processes, resulting in large economic losses, environmental pollution, and personnel injuries. In the year 2011, the company signed a cooperation agreement with Huazhong University of Science and Technology (HUST) to explore safety improvement measures. Finally, the research team from HUST developed the OFSM and the support system, and applied them in the company to improve the maintenance safety and efficiency. The performance of the OFSM system was examined during an oil-loading facility maintenance in the company.

\subsection{Off-site maintenance system implementation}

The equipment needed for repair was identified through the maintenance warning, as shown in Fig. 8. In this case study, two loading arms marked in red require maintenance, as displayed in Fig. 9. This figure shows a possible digital data interface for equipment management. It illustrates the equipment information (counterclockwise from the upper left), equipment classification, previous maintenance records, 3D model, basic attributes, and control information. Maintenance workers were assigned automatically based on previous maintenance records. The workers found the control valve, shut it down, and took emergency control measures under the guidance of a visualized maintenance control information, as depicted in Fig. 10. Other safety measures were taken subsequently to prepare for the dismantlement, as shown in Fig. 11. The workers got the visualized safety training (including the dismantling simulation) prior to conducting the dismantlement activity on site, as displayed in Fig. 12.

The faulty equipment (i.e., the two loading arms) were dismantled and labeled with the corresponding RFID tag. While conducting the maintenance operation in the workshop, the workers used an RFID reader to read the tag on each loading arm and obtained a rapid identification of the equipment and its information. The workers maintained a view of the loading arm and all the data and documents connected to it (Fig. 9). With the retrieval and access of these data, the workers easily captured the operating conditions (e.g., temperature, pressure, and heat insulation level). Besides, they can make the right decision on whether to repair or replace the equipment according to the maintenance information (e.g., data of production and service life). Furthermore, the workers had retrieved the

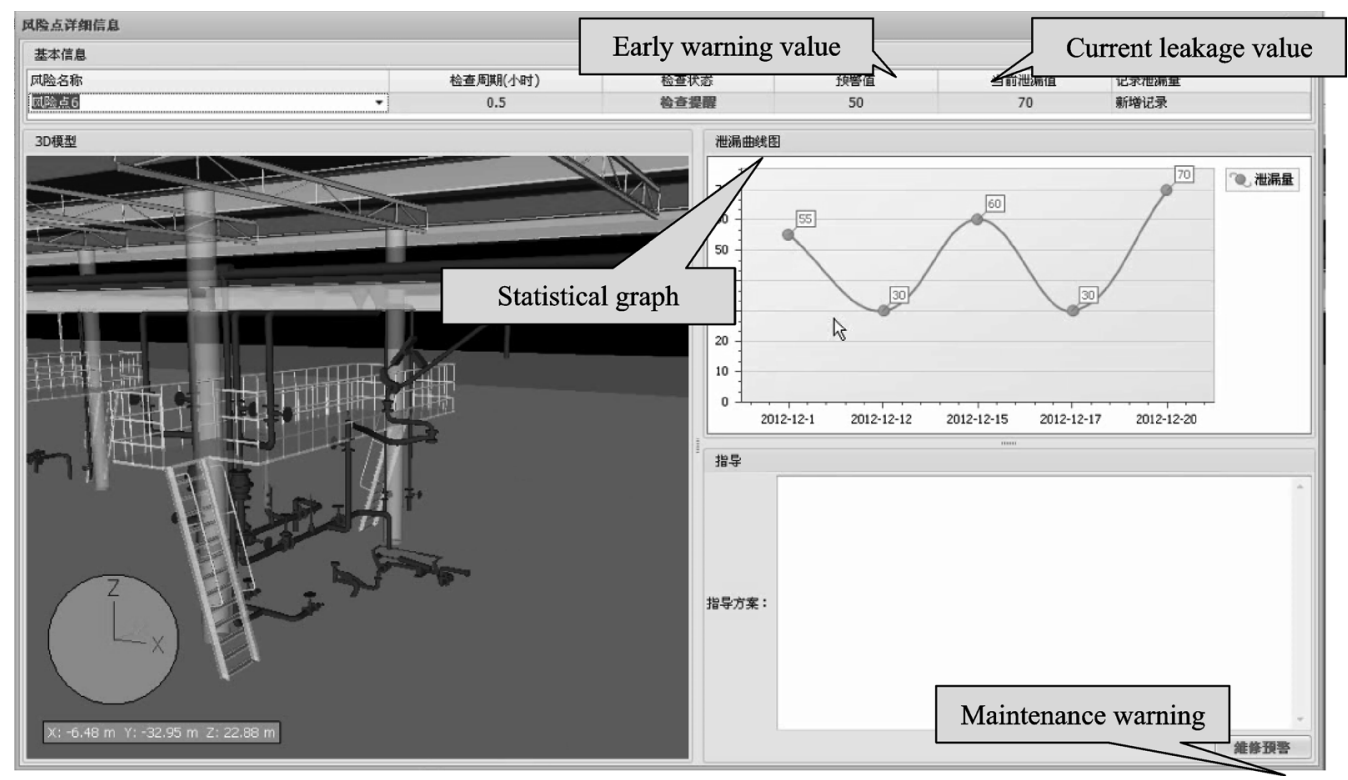

Fig. 8 Statistical analysis and maintenance warning 


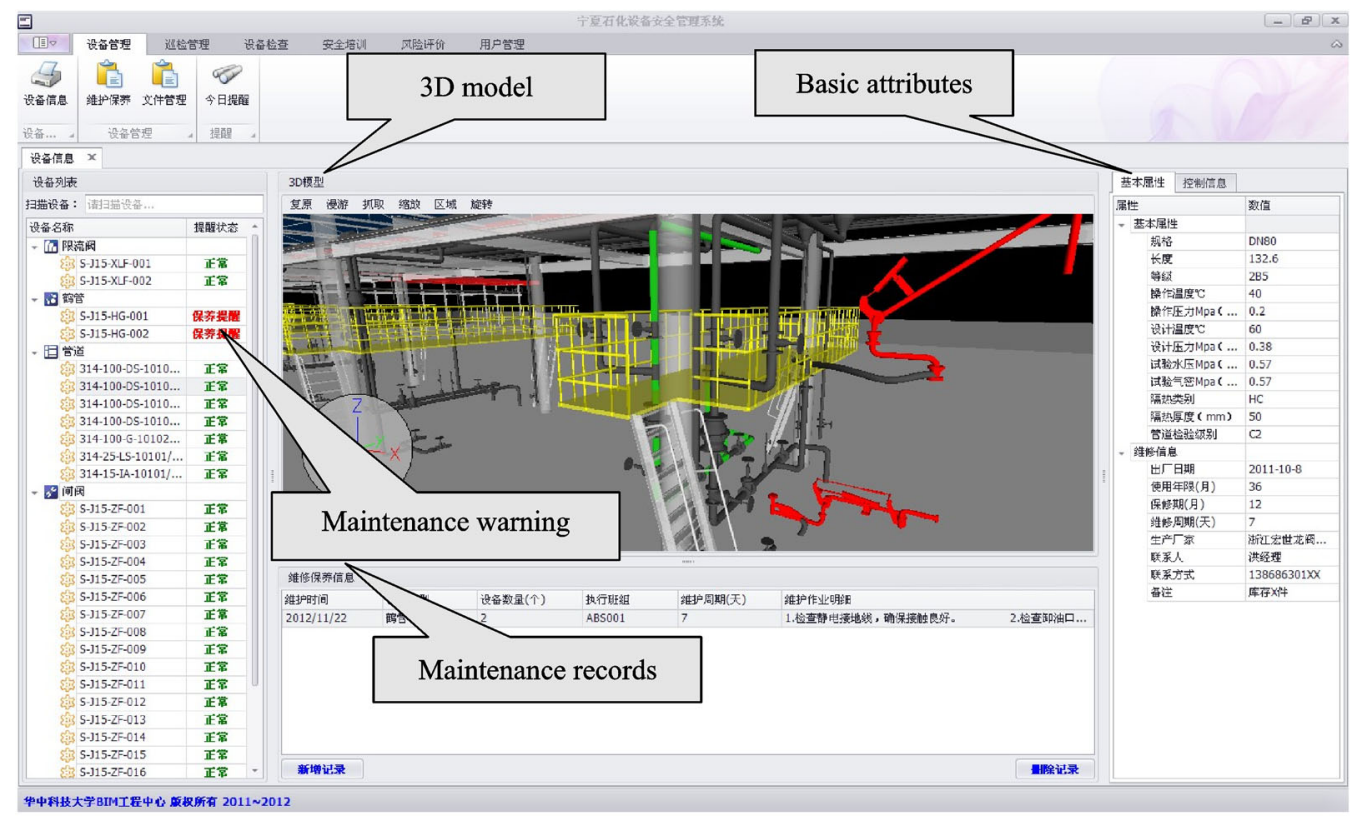

Fig. 9 Digital data interface of off-site maintenance system

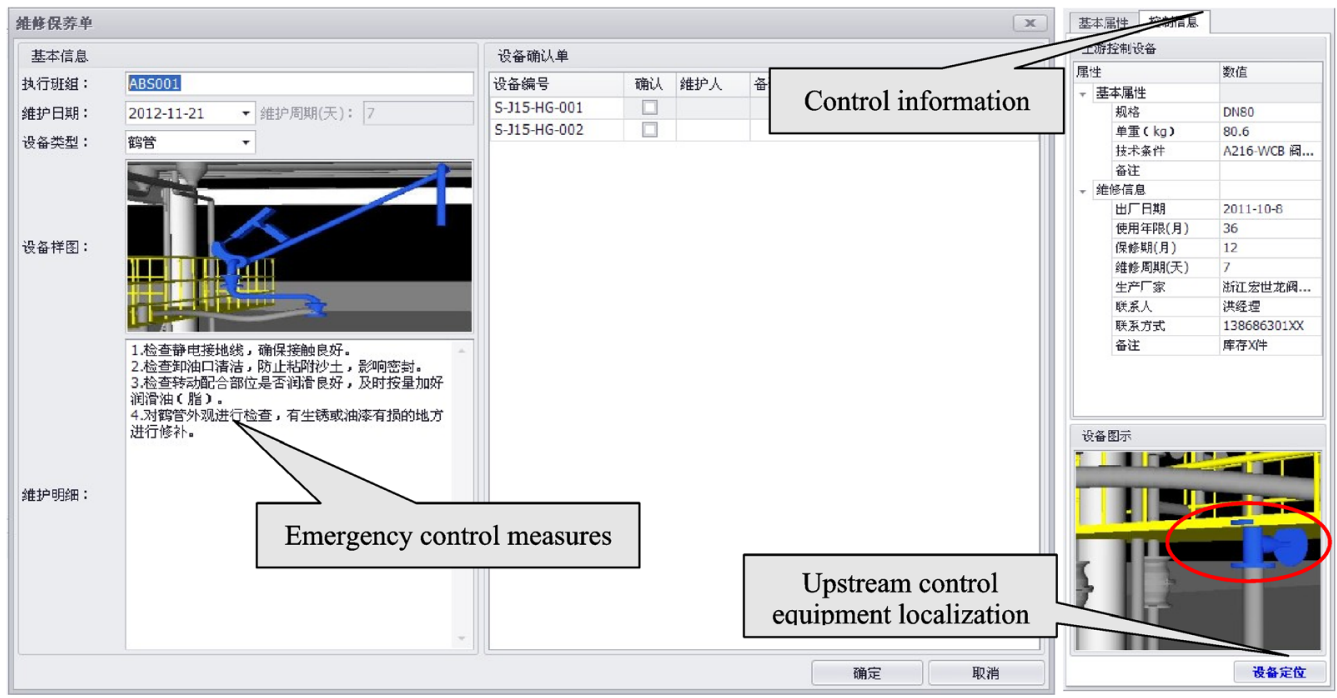

Fig. 10 Visualized maintenance control information

data from previous maintenance records, which contributed to better understanding of the equipment operating state.

The detection test was conducted according to the standards and requirements and the equipment was examined under practical production conditions as presented in the attributes information list (Fig. 9). After the faults had been eliminated, the installation team conducted the reinstallation operation. The tag on each loading arm brought the workers to the same 3D model and helped them to check the equipment's original location in the graphical interface, improving the efficiency of reinstallation.

\subsection{Discussion}

Conventional oil-loading facility maintenance takes the ONSM mode. However, accidents are prone to occur under the complicated and high-risk work environment on site. A risk assessment on the ONSM of an oil-loading facility is presented in Table 4, based on a series of interviews with safety experts and maintenance workers. When taking the mode of OFSM, the maintenance was undertaken under a controllable environment. A risk assessment on the OFSM of oil-loading facility is presented in Table 5. The risk classification criteria are shown in Table 6.

From these three tables, we can observe that OFSM 


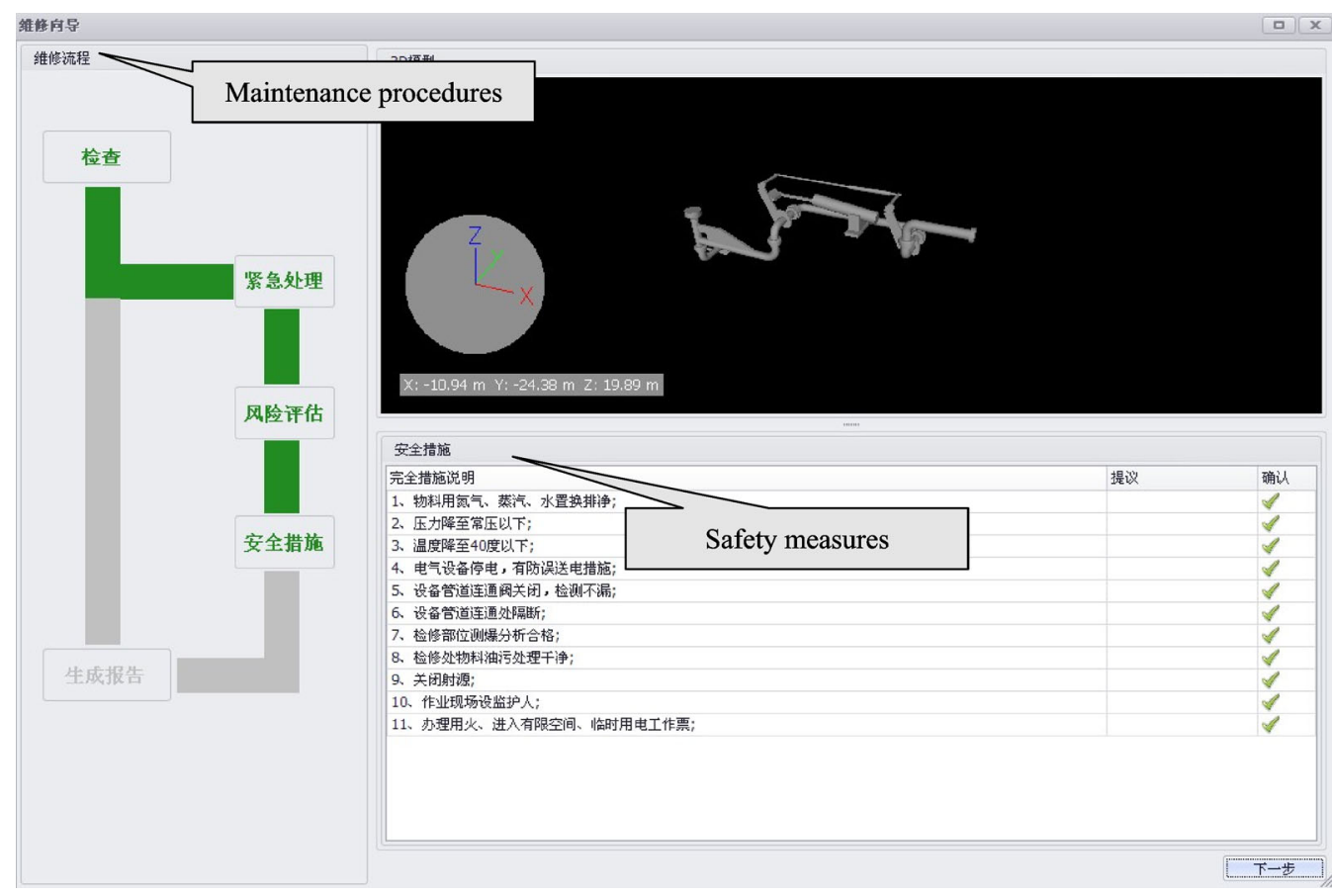

Fig. 11 Maintenance procedure direction

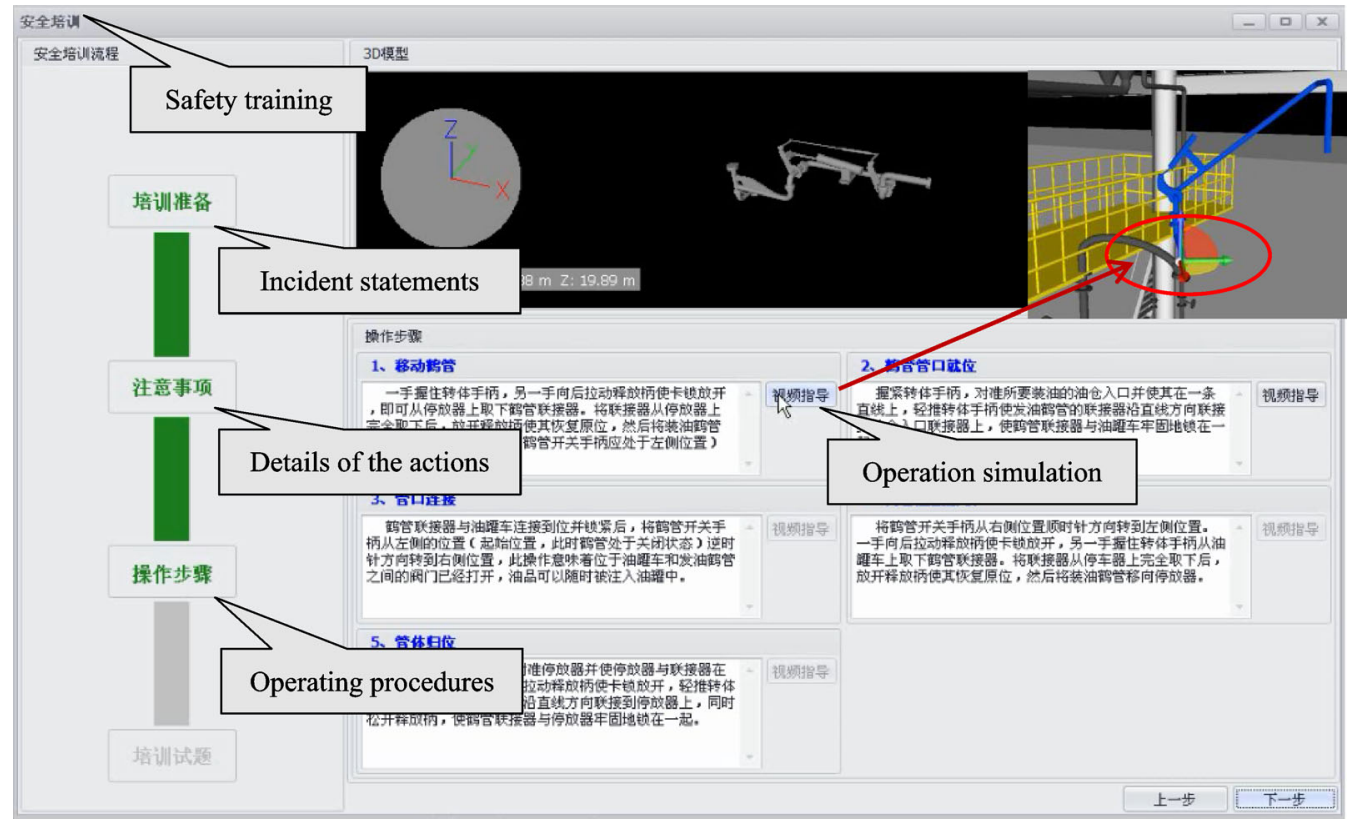

Fig. 12 Visualized safety training and dismantling simulation

resulted in risk reduction, not only on the risk types, but also on the risk levels. In ONSM, there are five types of risks and three of them are in high level. However, these five types of risks are eliminated when conducting OFSM. Instead, there are only two types of general risks in OFSM. The results indicated that OFSM can significantly improve the maintenance safety performance. It can be explained as follows:
(1) The volatilization of oil and gas cannot be completely isolated from the operating area in ONSM, and it is inevitable to produce sparks during the maintenance process. Therefore, long-time exposure to this risky environment may render the maintenance workers vulnerable to injury from fire accidents. However, in the OFSM workshop, the sparks produced during the maintenance process would have no effect on maintenance 
Table 4 Risk assessment on the ONSM of oil-loading facility

\begin{tabular}{|c|c|c|c|c|c|c|}
\hline \multirow{2}{*}{ Risk factor } & \multirow{2}{*}{ Risk type } & \multicolumn{4}{|c|}{ Risk assessment } & \multirow{2}{*}{ Risk level } \\
\hline & & $\mathrm{L}$ & $\mathrm{E}$ & $\mathrm{C}$ & LEC & \\
\hline Volatilization of oil and gas & Fire & 8 & 5 & 5 & 200 & 4 \\
\hline Flammable and explosive medium & Fire, explosion & 3 & 3 & 15 & 135 & 3 \\
\hline Toxic medium leak & Poisoning & 1 & 1 & 7 & 7 & 1 \\
\hline Disorderly tools & Object strikes & 7 & 5 & 3 & 105 & 3 \\
\hline Improper personnel positioning & Medium splash damage & 6 & 4 & 2 & 48 & 2 \\
\hline
\end{tabular}

Table 5 Risk assessment on the OFSM of oil-loading facility

\begin{tabular}{|c|c|c|c|c|c|c|}
\hline \multirow{2}{*}{ Risk factor } & \multirow{2}{*}{ Risk type } & \multicolumn{4}{|c|}{ Risk assessment } & \multirow{2}{*}{ Risk level } \\
\hline & & $\mathrm{L}$ & $\mathrm{E}$ & $\mathrm{C}$ & LEC & \\
\hline Transportation & Vehicle accidents & 4 & 3 & 3 & 36 & 2 \\
\hline Lifting & Crane accident & 3 & 4 & 5 & 60 & 2 \\
\hline
\end{tabular}

Table 6 Risk classification criteria

\begin{tabular}{lcc}
\hline LEC value & Risk level & Risk description \\
\hline$>320$ & 5 & Extremely risky, need to shut down \\
$160-320$ & 4 & Highly risky, need to immediately rectify \\
$70-160$ & 3 & Significantly risky, need to take some measures \\
$20-70$ & 2 & Generally risky, need to pay attention \\
$<20$ & 1 & Slightly risky, can be accepted \\
\hline
\end{tabular}

safety, as the volatilization of oil and gas can be isolated completely.

(2) The existing flammable, explosive, and toxic media in/around the processing installation expose the maintenance workers to an inherently risky environment. However, the risks from the flammable, explosive, and toxic medium leaks are eliminated when operating in the workshop.

(3) The materials, tools, and maintenance workers are crowded in a narrow operating area in ONSM, resulting in object strikes and medium splash damages. When conducting OFSM, well-organized maintenance materials and tools are provided in the workshop, and less maintenance workers are required to carry out maintenance works in a spacious area.

(4) OFSM may bring new risks during the transportation and lifting processes. However, these risks can be effectively controlled through conventional safety management measures.

Moreover, the efficiency and effectiveness of information retrieval were also improved in OFSM. First, traditional document preparation may take 20 to 30 minutes before the start of maintenance. The maintenance workers have to take time to identify the upstream control equipment on site. However, with the OFSM system, the acquisition of equipment identification and emergency control measures is automatically realized. Second, the information retrieval of various maintenance objects is time-consuming in the current paper documents or information systems, which generally took only a few seconds in this case study with the application of the RFID technology. Third, compared with the aid of conventional CAD drawing files and other paper documents, the 3D operation simulation facilitated the understanding of operating procedures and identification of disassembling/ assembling sequence while conducting the dismantling/ reinstallation operation.

\section{Conclusions}

This study proposed an OFSM system to improve the safety performance and efficiency. Two critical tasks were conducted: proposal of OFSM to solve the safety challenges in ONSM and development of a support system to ensure an efficient and effective implementation of OFSM. OFSM will shift the conduct of on-site operations to the OFSM workshop as much as possible, reducing the dangerous effects of unstable equipment, complicated work environment, and human error. The support system with the visualization, information management, and localization capabilities will provide instant accessibility of comprehensive information required for OFSM. The benefits of the proposed OFSM system were demonstrated through a practical maintenance operation of an oil-loading facility in Guangxi Petrochemical Company. The conclusions drawn from this study are as follows:

(1) The proposed OFSM can reduce operating risks. Five types of risks were eliminated during the OFSM of two loading arms of an oil-loading facility in the case study, including three significant risks. Under a controllable environment, by minimizing on-site operations, congestion, and reduction of exposure to hazards, the improvement of maintenance safety is realized in OFSM. 
(2) The developed OFSM system can improve operating efficiency. The visualization module facilitates the identification of maintenance requirements. It also provides maintenance workers with a virtual facility environment to help them obtain an overall understanding of the maintenance object. The information management module offers an accurate, complete, and consistent information. The localization module takes the workers to the right equipment and provides instant accessibility to information relating to that equipment by utilizing the RFID technology.

Future work includes (1) integrating an equipment maintenance decision-making module into the system to help in selecting the appropriate maintenance strategy (ONSM or OFSM), as some equipment may be not suitable for the OFSM mode (e.g., due to high cost); (2) a long-term survey to further validate the application performance of the OFSM system.

\section{References}

Adolfo C M, Heguedas A S (2002). Models for maintenance optimization: A study for repairable systems and finite time periods. Reliability Engineering \& System Safety, 75(3): 367-377

Attwood D, Khan F, Veitch B (2006). Can we predict occupational accident frequency? Process Safety and Environmental Protection, 84 (3): 208-221

Baesi S, Abdolhamidzadeh B, Hassan C, Hamid M D, Reniers G (2013). Application of a multi-plant QRA: A case study investigating the risk impact of the construction of a new plant on an existing chemical plant's risk levels. Journal of Loss Prevention in the Process Industries, 26(5): 895-903

Ben-Daya M, Duffuaa S O, Raouf A, Knezevic J, Ait-Kadi D (2009). Handbook of Maintenance Management and Engineering. London: Springer

Chien-Ho K (2009). RFID-based building maintenance system. Automation in Construction, 18(3): 275-284

Cong G P (2013). Study on intelligent decision-making of inspection and maintenance based on risk and condition for petrochemical equipment. Dissertation for the Doctoral Degree. Dalian: Dalian University of Technology (in Chinese)

Fabiano B, Curro F (2012). From a survey on accidents in the downstream oil industry to the development of a detailed near-miss reporting system. Process Safety and Environmental Protection, 90 (5): 357-367

Gibb A, Isack F (2003). Re-engineering through pre-assembly: Client expectations and drivers. Building Research and Information, 31(2): $146-160$

Guo L J, Gao J J, Yang J F, Kang J (2009). Criticality evaluation of petrochemical equipment based on fuzzy comprehensive evaluation and a BP neural network. Journal of Loss Prevention in the Process Industries, 22(4): 469-476

Hipkin I B, De C C (2000). TPM and BPR: Lessons for maintenance management. OMEGA-International Journal of Management
Science, 288-292

Ikuma L H, Nahmens I, James J (2011). Use of safety and lean integrated Kaizen to improve performance in modular homebuilding. Journal of Construction Engineering and Management, 137(7): 551-560

Ishekwene I V (2012). Improving the turnaround maintenance of the Escravos Gas Plant. Potchefstroom Campus, South Africa: NorthWest University

Jaillon L, Poon C S (2009). The evolution of prefabricated residential building systems in Hong Kong: A review of the public and the private sector. Automation in Construction, 18(3): 239-248

Knegtering B, Pasman H J (2009). Safety of the process industries in the 21 st century: A changing need of process safety management for a changing industry. Journal of Loss Prevention in the Process Industries, 22(2): 162-168

Konstandinidou M, Nivolianitou Z, Markatos N, Kiranoudis C (2006). Statistical analysis of incidents reported in the Greek Petrochemical Industry for the period 1997-2003. Journal of Hazardous Materials, 135(1-3): 1-9

Li G Z (2013). Research on Maintenance Strategy and Fault Diagnosis of Subway Train Based on RAMS, Dissertation for the Doctoral Degree, Beijing: Beijing Jiaotong University (in Chinese)

Li J R, Khoo L P, Tor S B (2006). Generation of possible multiple components disassembly sequence for maintenance using a disassembly constraint graph. International Journal of Production Economics, 102(1): 51-65

Liu G S (2003). Safety countermeasures for repairing petrochemical plants. Safety Health \& Environment, 3(8): 6-7 (in Chinese)

Luan X H (2011). BP US Texas refinery fire explosion. Modern Occupational Safety, 2011(10): 82-83 (in Chinese)

Maas G, van Eekelen B (2004). The Bollard- the lessons learned from an unusual example of off-site construction. Automation in Construction, 13(1): 37-51

Martorell S, Villanueva J F, Carlos S, Nebot Y, Sánchez A, Pitarch J L, Serradell V (2005). RAMS $+\mathrm{C}$ informed decision-making with application to multi-objective optimization of technical specifications and maintenance using genetic algorithms. Reliability Engineering \& System Safety, 87(1): 65-75

Moghadam M, Al-Hussein M, Al-Jibouri S, Telyas A (2012). Post simulation visualization model for effective scheduling of modular building construction. Canadian Journal of Civil Engineering, 39(9): 1053-1061

Motawa I, Almarshad A (2013). A knowledge-based BIM system for building maintenance. Automation in Construction, 29: 173-182

Murthy D N P, Asgharizadeh E (1999). Optimal decision making in a maintenance service operation. European Journal of Operational Research, 116(2): 259-273

Nivolianitou Z, Konstandinidou M, Kiranoudis C, Markatos N (2006). Development of a database for accidents and incidents in the Greek petrochemical industry. Journal of Loss Prevention in the Process Industries, 19(6): 630-638

Papazoglou I A, Nivolianitou Z, Aneziris O, Christou M D, Bonanos G (1999). Risk-informed selection of a highway trajectory in the neighborhood of an oil-refinery. Journal of Hazardous Materials, 67 (2): 111-144

Rodriguez D A, Norrish J, Nicholson A (2009). Robot programming for non repetitive repair operations using vision system. Trends in 
Welding Research, 671-675

Sherwin D (2000). A review of overall models for maintenance management. Journal of Quality in Maintenance Engineering, 6(3): 138-164

Singh V, Gu N, Wang X Y (2011). A theoretical framework of a BIMbased multi-disciplinary collaboration platform. Automation in Construction, 20(2): 134-144

Skogdalen J E, Vinnem J E (2012). Combining precursor incidents investigations and QRA in oil and gas industry. Reliability Engineering \& System Safety, 101: 48-58

Vagliasindi F (2003). Gestire la manutenzione: perché e come. FrancoAngeli

Waeyenbergh G, Pintelon L (2002). A framework for maintenance concept development. International Journal of Production Economics, 77(3): 299-313

Waeyenbergh G, Pintelon L (2009). CIBOCOF: A framework for industrial maintenance concept development. International Journal of Production Economics, 121(2): 633-640

Wang L, Chu J, Wu J (2007). Selection of optimum maintenance strategies based on a fuzzy analytic hierarchy process. International Journal of Production Economics, 107(1): 151-163

Wang Q Z (2003). Responsible Subject of Oil Pollution Damage Caused by Ship Collision and Its Responsibility, Dissertation for the Ph.D degree

Wong C K, Fung I, Tam C M (2010). Comparison of using mixed-integer programming and genetic algorithms for construction site facility layout planning. Journal of Construction Engineering and Management, 136(10): 1116-1128

Xue P L, Zeng W H (2010). Policy issues on the control of environmental accident hazards in China and their implementation. International Conference on Ecological Informatics and Ecosystem Conservation, 2(ISEIS 2010): 440-445

Yuan Z J, Guo Y (2011). Risk identification and control measures of overhaul key operation about natural gas purification equipment. Chemical Engineering of Oil \& Gas, 40(4): 429-432 (in Chinese)

Zhang C L (2012). HSE management for inspection and maintenance operations of petrochemical plants. Safety Health \& Environment, 12 (1): 54-56 (in Chinese)

Zhang D K, Ye Y C (2010). The unsafe factors and countermeasures in petrochemical equipment maintenance. Petrochemical Equipment, 13(4): 57-59 (in Chinese)

Zhao J S, Joas R, Abel J, Marques T, Suikkanen J (2013). Process safety challenges for SMEs in China. Journal of Loss Prevention in the Process Industries, 26(5): 880-886 